\title{
Definition of School Based Family Counseling and Therapy
}

Michael Stokely Kelly ${ }^{1}$

1 Loyola University Chicago

Note: Initial draft definition was co-created with Peter Gieger, LMFT \& Kathleen C. Laundy, PsyD, LMFT, MSW as part of the August 2017 Oxford Symposium in SBFC, Venice International University

SBFC\&T is both $[A]$ a model and $[B]$ a metamodel

A. School Based Family Counseling and Therapy, the model, is, in its most basic form, the application of family therapy principles and methods to boosting student resiliency and achievement in schools

B. School Based Family Counseling and Therapy, the metamodel, is a multi-systems approach to improving the lived experience of persons in relationship; the metamodel can be utilized by professionals-

- working in (or not in) a school setting

- where the symptom-bearer may (or may not be) a child alone-could be a parent, teacher, group or policymaker

- where the unit of treatment may (or may not) include a child

- where the SBFC\&T professional is a policymaker and the intervention is directed at the community

I. School Based Family Counseling and Therapy, the metamodel, is characterized and defined by the actions and stance of the professional in combatting the problem on behalf of the symptom-bearer and other stakeholders

II. The stakeholders are any persons identifiable as affected by the problem, ranging from the family and school system [as in A. the model] to the wider community [in B. the metamodel]

III. The symptom-bearer is any person identifiable as affected by the problem in a 
manner producing clinical symptoms that may be the focus of both concern and efforts in remediation; at the macro level an intervention in SBFC\&T may be designed to prevent or forestall symptoms in potential symptom-bearers [example: a professional development program for teachers on collaborative ways to help prevent substance abuse in a high school]

IV. The problem is the symptom or adverse syndrome or circumstance affecting the symptom-bearer [or potential symptom-bearer] and other stakeholders

V. The professional may be a member of a mental health or other helping profession (including legal, medical, education and policymaking) in which counseling and therapy is done with either primary or adjunctive professional purpose

VI. The stance of the professional is to:

(i) conceptualize the problem according to a multi-systems perspective

(ii) combat the problem and ameliorate the lived experience of symptom-bearer(s) and other stakeholders by

(iii) intervening creatively and efficiently to alter, mitigate or remove (or forestall) the environmental challenges experienced by the symptom-bearer(s)

VII. The actions of the professional in so doing:

(i) are efficient in that they secure the most useful systemic change with the least use of professional time spent [example: treating the parents' communication issues to relieve the pressure on the child in the home and secure improved behavior in the school, as compared with leaving the parents' issues untreated and giving supportive counseling to the child]

(ii) are creative in that they may include doing interventions which have not been done before or which have not been done before in quite this manner [example: a nonprofit organization commissions a panel of clinicians, neuroscientists and market researchers to create an innovative, accessible and nonpathological "story" about mental health and recovery that assists individuals to acquire resiliency, improve behavior and change more easily and quickly than would otherwise be the case] 